\title{
XMM-Newton discovery of soft X-ray absorption in the high-z superluminous Blazar RBS $315^{\star}$
}

\author{
E. Piconcelli and M. Guainazzi
}

European Space Astronomy Center (ESA), Apartado 50727, 28080 Madrid, Spain

e-mail: Enrico.Piconcelli@sciops.esa.int

Received 18 May 2005 / Accepted 14 September 2005

\section{ABSTRACT}

We present the analysis and the results of a $20 \mathrm{ks} X M M$-Newton observation of the extremely X-ray loud $\left(L_{\mathrm{X}} \approx 5 \times 10^{47} \mathrm{erg} \mathrm{s}^{-1}\right)$ flatspectrum radio quasar RBS 315 at a redshift of 2.69. This EPIC observation has allowed us to strongly constrain the slope of the continuum $(\Gamma=1.23 \pm 0.01)$ as well as to discover the presence of a sharp drop below $\approx 2 \mathrm{keV}$ in its spectrum. Such a flat photon index and the huge luminosity suggest that the X-ray emission is due to the low energy tail of the Comptonized spectrum, produced from plasma in a relativistic jet oriented close to our line of sight. Even though the hypothesis of a break in the continuum cannot be completely discarded as an explanation of the soft X-ray cutoff, the presence of intrinsic absorption appears more plausible. Spectral fits with cold $\left(N_{\mathrm{H}}^{z}=1.62_{-0.09}^{+0.09} \times 10^{22} \mathrm{~cm}^{-2}\right)$ and lukewarm $\left(N_{\mathrm{H}}^{z}=2.2_{-0.3}^{+0.9} \times 10^{22} \mathrm{~cm}^{-2} ; \xi=15_{-12}^{+38} \mathrm{erg} \mathrm{cm}^{-2} \mathrm{~s}^{-1}\right)$ absorbers are statistically indistinguishable. Remarkably, our results are very similar to those reported so far for other absorbed high- $z$ Blazars observed by XMM-Newton. The existence of this "homogeneous" class of jet-dominated superluminous obscured QSOs at high $z$ therefore could be important in the context of the formation and cosmological evolution of radio-loud objects.

Key words. galaxies: active - quasar: general - X-ray: individual: RBS 315

\section{Introduction}

According to Active Galactic Nuclei (AGNs) Unified models (Urry \& Padovani 1995) a relativistic jet emitting a beamed non-thermal continuum is present in radio-loud quasars (QSOs): if the jet points along our line of view, the QSO is seen as a Blazar. Due to strong amplification and collimation in the observer's frame Blazars appear as the most luminous objects in the sky from radio through gamma-rays. As pointed out by Fossati et al. (1998) the X-ray spectrum of Blazars hardens with increasing luminosity: in fact flat-spectrum radio-loud QSOs (FSRQs), which represent the most luminous class of Blazars, typically show photon indices $\Gamma \approx 1.3-1.5$ in the $2-10 \mathrm{keV}$ band (e.g. Boller et al. 2000; Donato et al. 2005; Ferrero \& Brinkmann 2003, F03 hereafter) i.e. flatter than usually observed in "normal" radio-loud $\left(\left\langle\Gamma_{2-10}\right\rangle \approx 1.75\right.$; Sambruna et al. $1999)$ or radio-quiet QSOs $\left(\left\langle\Gamma_{2-10}\right\rangle \approx 1.9\right.$; Piconcelli et al. 2005a).

The discovery of a low-energy cutoff due to heavy cold absorption $\left(\sim 10^{22-23} \mathrm{~cm}^{-2}\right)$ in the X-ray spectra of some luminous high- $z$ FSRQs (Elvis et al. 1994; Cappi et al. 1997; Boller et al. 2000; Yuan et al. 2000) was therefore unexpected since our line of sight should not intercept the obscuring torus.

* Based on observations with XMM-Newton, an ESA Science Mission with instruments and contributions directly funded by ESA Member states and the USA (NASA).
An explanation in terms of obscuration associated with intervening systems was promptly ruled out by O'Flaherty \& Jakobsen (1997) and Fiore et al. (1998) on the basis of statistical arguments, supporting therefore the hypothesis that the absorber is physically associated to the QSO. However, the properties of the absorbing matter are still poorly known and the observational constraints about its nature are very sparse. Unfortunately the low sensitivity and limited soft X-rays bandpass of ASCA and BeppoSAX data as well as the usual high $z$ of the "obscured" FSRQs prevented deeper investigations about the origin of the absorption features. Thanks to its unprecedented large collecting area, XMM-Newton has allowed the investigations in this field to be re-opened. Worsley et al. (2004a, W04 hereafter) claimed for a warm absorber in the spectrum of the Blazar PMN J0525-3343, while F03 found that both a warm and a cold absorber can fit the data of the Blazar PKS 2126158. On the other hand, some recent works based on $X M M$ Newton observations (F03; Grupe et al. 2004; Piconcelli et al. 2005b) do not confirm earlier ASCA measurement of a strong absorption toward three high- $z$ QSOs.

In this letter we present the analysis of the XMMNewton observation of RBS 315 that has allowed to explore in detail the $0.3-12 \mathrm{keV}$ spectrum of this source for the first time. RBS 315 was discovered as a powerful flat-spectrum radio source $\left(S_{6 \mathrm{~cm}}=595 \mathrm{mJy} ; \alpha_{6-20 \mathrm{~cm}}=0.2\right)$ by Lawrence et al. (1983) and subsequently identified as a QSO at $z=2.69$ with a 
radio-loudness parameter $R=S_{6 \mathrm{~cm}} / F_{\mathrm{B}} \approx 6500$ in the ROSAT Bright Survey (RBS; Schwope et al. 2000). RBS 315 was detected in X-rays by ROSAT and RXTE during the All-Sky Slew Survey (XSS; Sazonov et al. 2004). Both X-ray measurements imply a huge luminosity of $L_{\mathrm{X}} \gtrsim 10^{47} \mathrm{erg} \mathrm{s}^{-1}$ and a very flat hardness ratio, which is a clear indication of a convex spectrum.

\section{Observation and data reduction}

The XMM-Newton observation of RBS 315 took place on 2003 July 25 and it was performed with the EPIC PN (Struder et al. 2001) and MOS (Turner et al. 2001) cameras operating in full-frame mode. Data were reduced with $S A S 6.1$ (Loiseau et al. 2004), using the most updated calibration files available as of March 1, 2005. X-ray events corresponding to patterns $0-12(0-4)$ for the $\operatorname{MOS}(P N)$ cameras were selected. The event lists were filtered to ignore periods of high background flaring according to the method presented in Piconcelli et al. (2004) based on the cumulative distribution function of background lightcurve count-rates. After this data cleaning, we obtained as net exposure time 18.3, 21.5, $21.7 \mathrm{ks}$ for $P N, M O S 1$ and MOS2, respectively. The centroid position of RBS 315 in the EPIC image is RA $=02^{\mathrm{h}} 25^{\mathrm{m}} 04.5$, Dec $=+18^{\circ} 46^{\prime} 51^{\prime \prime}, 3.6^{\prime \prime}$ away from the position of its radio counterpart (Beasley et al. 2002). This shifting is, however, smaller than the astrometric error box radius of $X M M$-Newton $\left(\approx 6^{\prime \prime}\right)$. Source photons were extracted using a circular region of radius 36 arcsec centered at the source position while backgrounds were estimated from source-free similar regions close to the source on the same CCD. No exposure is affected by pile-up. Response matrices and ancillary response files were generated using the RMFGEN and ARFGEN tools in the $S A S$ software. Since difference between the MOS1 and MOS2 response matrices are a few percent, we created a combined MOS spectrum and response matrix. The $P N$ and $M O S$ spectra were then fitted simultaneously. Both spectra were grouped to give a minimum of 30 counts per bin in order to apply $\chi^{2}$ statistics. Given the current calibration uncertainties and the detector sensitivities (Kirsch et al. 2004), events outside the $0.3-12 \mathrm{keV}$ range were ignored in the $P N$ spectrum, while, for the MOS, we retained the $0.8-10 \mathrm{keV}$ band. The quoted errors on the model parameters correspond to a $90 \%$ confidence level for one interesting parameter $\left(\Delta \chi^{2}=2.71\right.$; Avni 1976). All luminosities are calculated assuming a $\Lambda \mathrm{CDM}$ cosmology with $\left(\Omega_{\mathrm{M}}, \Omega_{\Lambda}\right)=(0.3,0.7)$ and a Hubble constant of $70 \mathrm{~km} \mathrm{~s}^{-1} \mathrm{Mpc}^{-1}$.

\section{Results}

All the spectral fits were performed with the XSPEC package (version 11.3.0). The models presented in this section include an absorption component due to the line-of-sight Galactic column of $N_{\mathrm{H}}=10^{21} \mathrm{~cm}^{-2}$ (Dickey \& Lockman 1990). We initially fitted the hard (2-10 keV, corresponding to $7.4-37 \mathrm{keV}$ in the frame of RBS 315) portion of the spectrum with a power law. This fit turned out to be very good yielding an associated $\chi_{\text {red }}^{2}=1.07$ with a resulting best-fit value of the photon index $\Gamma=1.24_{-0.02}^{+0.01}$. Visual inspection of the datato-model ratio residuals did not suggest the presence of any

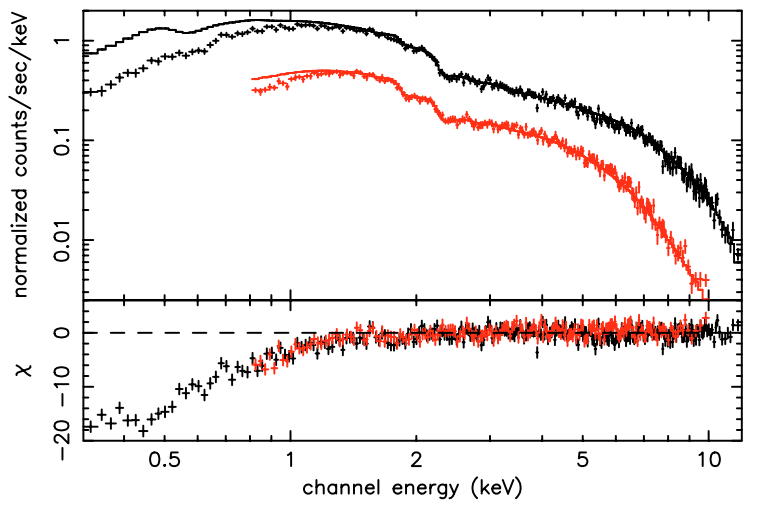

Fig. 1. Continuum power-law fit to the hard band of the $P N$ (top) and MOS (bottom) spectra of RBS 315 extrapolated over the $0.3-12(0.8-10) \mathrm{keV}$ band for $P N(M O S)$. The lower panel shows the deviations of the observed data from the model in unit of standard deviations.

emission/absorption features. However, the extrapolation of the power law to energies lower than $2 \mathrm{keV}$ clearly revealed the presence of a deep deficit in the soft range of the spectrum (Fig. 1). Fitting the data over the $0.3-12 \mathrm{keV}$ band with a simple power law (Model PL) yielded a $\chi^{2}$ (d.o.f.) 1821(455). We accounted for the soft X-ray spectral drop by means of a neutral ("cold") absorption component in the fitting model. This fit (model APL hereafter) resulted in a dramatic statistical improvement i.e. at the $>99.99 \%$ confidence level according to an $F$-test once compared with the model PL $\left(\Delta \chi^{2}=1342\right)$, with a $\chi^{2}$ (d.o.f.) $=479(454)$. This fit therefore provides an excellent parametrization of the X-ray spectrum of this Blazar with a best-fit value of $\Gamma=1.23 \pm 0.01$ and $N_{\mathrm{H}}^{z}=1.62_{-0.09}^{+0.09} \times 10^{22} \mathrm{~cm}^{-2}$ for the spectral index and the intrinsic column density of the "cold" absorber, respectively. Using Model APL we measured a flux of $F_{0.5-2}=3.1 \times 10^{-12} \mathrm{erg} \mathrm{cm}^{-2} \mathrm{~s}^{-1}$ and $F_{2-10}=$ $1.6 \times 10^{-11} \mathrm{erg} \mathrm{cm}^{-2} \mathrm{~s}^{-1}$ in the low $(0.5-2 \mathrm{keV})$ and high $(2-10 \mathrm{keV})$ energy bands, respectively. After correction for both Galactic and intrinsic absorption column densities, these correspond to luminosities of $L_{0.5-2}=7.5 \times 10^{46} \mathrm{erg} \mathrm{s}^{-1}$ and $L_{2-10}=3.5 \times 10^{47} \mathrm{erg} \mathrm{s}^{-1}$, respectively. It is worth to note that if the redshift of the absorber if fixed to $z=0$, the resulting column density value decreases to $N_{\mathrm{H}}(z=0)=9.4 \pm 0.1 \times 10^{20} \mathrm{~cm}^{-2}$. However this fit, with an associated $\chi^{2}$ (d.o.f. $)=508(454)$, is statistically worse than Model APL (i.e. $\Delta \chi^{2}=29$ for the same number of d.o.f.) and some residuals, as ratio between model and data, are clearly present in the soft X-ray band. The hypothesis that the absorber could be associated with our Galaxy appears, therefore, quite unlikely.

As W04 suggested the presence of a warm absorber as the most plausible explanantion of the soft X-ray cutoff in the XMM-Newton spectrum of the Blazar PMN J0525-3343 at $z=4.4$, we then investigated if it is the case also for RBS 315 . This fit, performed using the ABSORI model in XSPEC, is statistically equivalent to that with the APL model, i.e. associated $\chi^{2}$ (d.o.f. $)=478(453)$. The resulting column density of the warm gas was $N_{\mathrm{H}}=2.2_{-0.3}^{+0.9} \times 10^{22} \mathrm{~cm}^{-2}$, while the best-fit value ionization parameter was $\xi=15_{-12}^{+38} \mathrm{erg} \mathrm{cm}^{-2} \mathrm{~s}^{-1}$. This value is comparable to what reported by W04. 


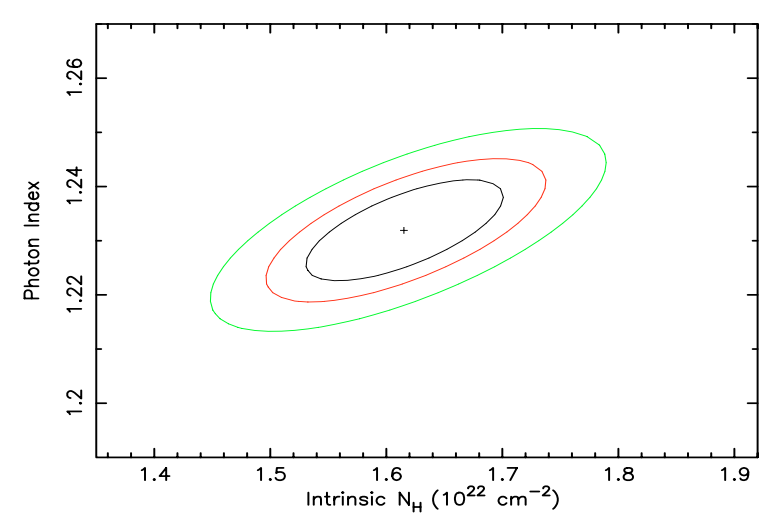

Fig. 2. Confidence contour plot showing the photon index against the rest-frame column density $\left(N_{\mathrm{H}}^{z}\right)$ of the absorber. The contours are at $68 \%, 90 \%$ and $99 \%$ confidence levels, respectively, for two interesting parameters.

An alternative explanation for the convex spectrum in Blazar is the presence of an underlying break in the continuum. Fossati et al. (1998) have shown that the spectral energy distribution of Blazars is "double-humped" with two main components due to the synchrotron emission and Inverse Compton (IC) scattering of relativistic electrons off synchrotron or ambient "seed" photons, respectively. In the case of the FSRQs, such as RBS 315 , the IC hump peaks at $\sim 10-100 \mathrm{MeV}$ : this means that the spectrum in the XMM-Newton bandpass is dominated by the Comptonized "boosted" emission from the jet. A spectral break can originate through a low-energy cutoff in the energy distribution of the radiating electrons or by a sharply peaked external "seed" photon distribution (e.g. Fabian et al. 2001). We then tried an alternative fit modeling the spectrum with a broken power law. This parameterization yielded unacceptable fit with an associate $\chi^{2}$ (d.o.f.) $=544(453)$, i.e. significantly worse than that derived with model APL. A model with two (unabsorbed) power laws also turned out in a very poor fit $\left(\chi_{\text {red }}^{2} \gtrsim 3\right)$.

\section{Discussion}

The EPIC observation presented here provides the first goodquality X-ray spectrum of RBS 315 . It has allowed us to strongly constrain the slope of the continuum $(\Gamma=1.23 \pm 0.01)$ as well as to discover the presence of a sharp drop in the observed spectrum below $1.5 \mathrm{keV}$. Such a value of photon index is consistent with previous X-ray observations of luminous FSRQs $\left(\left\langle\Gamma_{0.3-4.5 \mathrm{keV}}\right\rangle=1.48\right.$, Sambruna et al. 1997; see also W04 and F03) and is also in agreement with the trend found by Fossati et al. (1998) for a large sample of Blazars according to which the more X-ray luminous the object is, the flatter X-ray spectrum it possesses. The explanation for this behavior is that the X-ray continuum in FSRQs is believed to be the low energy tail of the Comptonized spectrum peaking in the $\mathrm{MeV}-\mathrm{GeV}$ range and as the peak moves to lower energies with increasing luminosity, the X-ray spectrum therefore becomes harder.

The most important results of our analysis is the discovery of a soft X-ray spectral flattening in RBS 315. In the previous section we tested three different models in order to account for this spectral feature: a power law modified by either a (i) cold or a (ii) warm absorption component and (iii) a broken power law. The latter provided the worst description of the XMMNewton spectrum with an associated $\chi_{\text {red }}^{2}=1.2$. Furthermore, as pointed out by Worsley et al. (2004b), an explanation of the observed cutoff in terms of a spectral break is not particularly compelling since such a feature is not observed so far in any of the nearby FSRQs, for which high-quality X-ray data have been collected. An additional problem that further weakens this scenario is the sharpness of the observed break as it takes place over a energy range of a few keVs in the QSO frame. It would therefore require an unlikely, nearly monochromatic "seed" photon distribution (e.g. Ghisellini 1996, for details) to be generated.

Hence, even though the hypothesis of a break in the continuum cannot be completely discarded, it appears quite unlikely once compared with the possible presence of absorption in this FSRQ.

On the other hand, the spectral fittings with cold and warm absorber are statistically indistinguishable: using the present $\mathrm{X}$-ray data it is not possible to constrain the ionization state of the absorbing matter better than $\xi=15_{-12}^{+38} \mathrm{erg} \mathrm{cm}^{-2} \mathrm{~s}^{-1}$. Moreover, the most prominent hallmarks of a warm absorber, typically the Fe M-shell UTA and the O/Ne absorption edges, are located in the rest-frame energy interval $0.6-0.9 \mathrm{keV}$ which is, unfortunately, outside the observed (i.e. $0.3-12 \mathrm{keV}$ ) band due to the high $z$ of the source. Furthermore the paucity of information about the characteristics of RBS 315 at other wavelengths in the literature does not allow to put any additional useful constraints on the nature of the absorber. However, the X-ray constraint on the ionization status matches well with those reported so far for other warm absorbers possibly detected in high-z Blazars (e.g. W04; F03; Worsley et al. 2004b) where the obscuring matter was found to be nearly neutral or with loosely constrained $\xi \lesssim 50 \mathrm{erg} \mathrm{cm}^{-2} \mathrm{~s}^{-1}$. The inferred column density of the absorbing material is $N_{\mathrm{H}} \approx$ a few $\times$ $10^{22} \mathrm{~cm}^{-2}$. Remarkably, such a value is very similar to those reported from XMM-Newton observations of all the other obscured Blazars at high $z$ for which, bearing in mind the small number statistics, the measured column densities of the absorbers seem to narrowly cluster around $N_{\mathrm{H}} \approx 10^{22} \mathrm{~cm}^{-2}$ (e.g. F03; W04; Yuan et al. 2005; Grupe et al 2005). $N_{\mathrm{H}}$ values $\lesssim 10^{22} \mathrm{~cm}^{-2}$ are, however, hard to detect at high redshifts and, hence, a selection effect is likely present.

Soft-energy cut-offs are more common in radio-loud objects (Fiore et al. 1998). One may therefore speculate that a mechanism of collisional ionization might be at work by multiple shocks along the jet (e.g. Gupta et al. 2005). A similar phenomenon is believed to be present in the NLR of nearby Seyfert galaxies which are spatially associated with their radio jet structure, suggesting that the NLR originates from the compression due to the interaction between the outflowing radio material and the ambient gas in the galaxy (Capetti et al. 1996). As the Blazar X-ray emission is dominated by relativistically beamed components from the jet, it is very likely that the obscuration may be due to jet-linked material and physical processes. 
Our result strengthens the evidence for the existence of a population of superluminous Blazar-like AGNs at $z>2$ which show soft X-ray cutoffs due to the likely presence of large amounts of intrinsic absorbing matter with similar properties. The discovery of these jet-dominated superluminous (i.e. $L_{0.5-10} \gtrsim 10^{47} \mathrm{erg} \mathrm{s}^{-1}$ ) obscured QSOs at high $z$ is also important in the context of the formation and cosmological evolution of radio-loud objects. Soft X-ray spectral cutoffs seems in fact to be a prerogative of radio-loud QSOs (Fiore et al. 1998): could the gaseous environments in powerful Blazars be different from that in radio-quiet AGNs? Is the absorber intimately linked with the presence of relativistic jets?

Important clues about the physical state and geometry of the absorber would be given by more sensitive ultra-soft X-ray observations, for instance carried out by the under-study ESA XEUS satellite (e.g. Bleeker \& Mendez 2002). Furthermore, more robust constraints on the ionization state of the obscuring matter may be also achieved with optical and UV observations by the detection of spectral features typical of an ionized absorber.

Acknowledgements. We thank the anonymous referee for a valuable report. We are grateful to the staff members of the $X M M$ Newton Science Operations Center for their support. E.P. thanks P. Rodriguez and J. A. Carter for helpful discussions.

\section{References}

Avni, Y. 1976, ApJ, 210, 642

Beasley, A. J., Gordon, D., Peck, A., et al. 2002, ApJS, 141, 13

Bleeker, J., \& Mendez, M. 2002, Proc. Symp. New Visions of the $\mathrm{X}$-ray Universe in the XMM-Newton and Chandra Era, ESA SP-488 [arXiv: astro-ph/0207283]

Boller, T., Fabian, A. C., Brandt, W. N., \& Freyberg, M. J. 2000, MNRAS, 315, 23
Capetti, A., Axon, D. J., Macchetto, F., et al. 1996, ApJ, 469, 554

Cappi, M., Matsuoka, M., Comastri, A., et al. 1997, ApJ, 478, 492

Dickey, J. M., \& Lockman, F. J. 1990, ARA\&A, 28, 215

Donato, D., Sambruna, R. M., \& Gliozzi, M. 2005, A\&A, 433, 1163

Elvis, M., Fiore, F., Wilkes, B. J., \& McDowell, J. 1994, ApJ, 422, 60

Fabian, A. C., Celotti, A., Iwasawa, K., et al. 2001, MNRAS, 323, 373

Ferrero, E., \& Brinkmann, W. 2003, A\&A, 402, 465 (F03)

Fiore, F., Elvis, M., Giommi, P., \& Padovani, P. 1998, ApJ, 429, 79

Ghisellini, G. 1996, Proc. Gamma-ray Emitting AGN, 71 [arXiv:astro-ph/9612042]

Grupe, D., Mathur, S., Wilkes, B., \& Elvis, M. 2004, ApJ, 127, 1

Grupe, D., Mathur, S., Wilkes, B., \& Osmer, P. 2005, AJ, submitted [arXiv: astro-ph/0501521]

Gupta, N., Srianand, R., \& Saikia, D. 2005, MNRAS, 316, 451

Kirsch, M., Alteri, B., Chen, B., et al. 2004, SPIE, 5488, 103

Lawrence, C. R., Bennett, C. L., Garcia-Barreto, J. A., et al. 1983, ApJS, 51, 67

Loiseau, N. 2004, XMM-Newton Science Analysis Users Guide v3.1

O'Flaherty, K. S., \& Jakobsen, P. 1997, ApJ, 479, 673

Piconcelli, E., Jimenez-Bailon, E., Guainazzi, M., et al. 2004, MNRAS, 351, 161

Piconcelli, E., Jimenez-Bailon, E., Guainazzi, M., et al. 2005a, A\&A, 432, 15

Piconcelli, E., Guainazzi, M., Cappi, M., et al. 2005b, A\&A, 432, 835

Schwope, A., Hasinger, G., Lehmann, I., et al. 2000, Astron. Nachr., 321,1

Struder, L., Briel, U., Dennerl, K., et al. 2001, A\&A 365, L18

Turner, M. J. L., Abbey, A., Arnaud, M., et al. 2001, A\&A, 365, L27

Urry, C. M., \& Padovani, P., 1995, PASP, 107, 803

Worsley, M. A., Fabian, A. C., Turner, A. K., et al. 2004a, MNRAS, 350, 207 (W04)

Worsley, M. A., Fabian, A. C., Celotti, A., \& Iwasawa, K. 2004b, MNRAS 350, 67

Yuan, W., Matsuoka, M., Wang, T., et al. 2000, ApJ, 545, 625

Yuan, W., Fabian, A. C., Celotti, A., et al. 2005, MNRAS, 358, 432 